\title{
THE FREQUENCY DECOMPOSITION MULTILEVEL METHOD: A ROBUST ADDITIVE HIERARCHICAL BASIS PRECONDITIONER
}

\author{
ROB STEVENSON
}

\begin{abstract}
Hackbusch's frequency decomposition multilevel method is characterized by the application of three additional coarse-grid corrections in parallel to the standard one. Each coarse-grid correction was designed to damp errors from a different part of the frequency spectrum. In this paper, we introduce a cheap variant of this method, partly based on semicoarsening, which demands fewer recursive calls than the original version. Using the theory of the additive Schwarz methods, we will prove robustness of our method as a preconditioner applied to anisotropic equations.
\end{abstract}

\section{INTRODUCTION}

As is well known, the rate of convergence of a multilevel method applied to a discretized elliptic boundary value problem is less than one uniformly in the top level. Yet, without a special choice of the components of the method, the rate of convergence tends to one as the problem becomes less elliptic (singularly perturbed problems), that is, the method is not robust. This paper concentrates on the question of robustness for so-called anisotropic problems. The classical way to obtain a robust multilevel method is to choose a smoother adapted to the problem. A disadvantage of this approach is that the resulting smoothers are often expensive, not well parallelizable or, in three dimensions, hard to find.

An alternative approach is to add more coarse-grid corrections to the multilevel method. Representatives of this class of methods are Hackbusch' Frequency Decomposition Multilevel Method (FDMLM) ([2,3,5,6]), which is the subject of this paper, and the Multiple Semi-Coarsened Grids Method ([8, 9, 10]) introduced by Mulder.

In two dimensions, the FD Two-Level Method consists of four coarse-grid corrections, that can be performed in parallel, each of them designed to reduce errors in a (non-overlapping) part of the frequency spectrum. To speed up convergence, smoothers can be added to the algorithm but we shall not consider this option. In the (V-cycle) FDMLM, each of the four coarse-grid problems is solved by means of a recursive call, thus involving four coarse-grid corrections on the next coarser level. For a complete explanation of the ideas behind this method, we refer to the papers of Hackbusch. In [5], it has been proved that the FDTLM yields a

Received by the editor August 1, 1994.

1991 Mathematics Subject Classification. Primary 65N55, 65N30.

Key words and phrases. Frequency decomposition, multilevel method, semicoarsening, finite elements, hierarchical basis, additive Schwarz method, subspace decomposition, robustness. 
robust preconditioner, that is, the condition number of the preconditioned system is bounded uniformly in the top level and the anisotropy. Until now, robustness of the FDMLM is an open problem.

In this paper, we study a cheap variant of the FDMLM. As was already noted in [3], one of the coarse-grid problems generated by the FDTLM has a bounded condition number $\left(:=\lambda_{\max } / \lambda_{\min }\right)$ uniformly with respect to the level and the anisotropy. Therefore, instead of applying a recursive call, this system can better be solved using a cheap iterative solver as, e.g., Jacobi's method. Apart from this, with our FDMLM, we solve two of the three remaining coarse-grid problems by means of only two instead of four coarse-grid corrections on the next coarser level by using semicoarsening. It will appear then that also one of these two corrections yields a system with bounded condition number, which therefore can be solved cheaply. On the other system we apply the semicoarsening idea recursively.

For any dimension $d$, the complexity of the resulting algorithm is equivalent to the number of unknowns, even if one would apply more than one recursive calls at certain places in the algorithm. Considered as an additive Schwarz method or, in the terminology of [12], a Parallel Subspace Correction method, it consists of (\#levels) ${ }^{d}$ subspace corrections compared to $\sim\left(2^{d}\right)^{\# \text { levels }}$ subspace corrections for the FDMLM in its original form.

Using the theory of the additive Schwarz methods, we will prove robustness of our FDMLM as a preconditioner. To do that, we first reformulate the method in an abstract finite element context. This kind of formulation of a multilevel method was introduced in [1]. Then with the help of tensor products, the question of robustness will be reduced to the question of convergence of the method in one dimension applied to the identity and the Laplace operator.

In one dimension, the subspace decomposition that defines our method appears to be very similar to the decomposition of the finite element space into the differences of successive $L^{2}$-orthogonal projections onto the finite element spaces corresponding to coarser grids. In particular, we will show that also our decomposition induces an $L^{2}$-equivalent norm, which means convergence for the identity. The fact that the decomposition using $L^{2}$-orthogonal projections yields an $H^{1}$-equivalent norm plays a crucial role in the modern regularity-free convergence proofs of standard multilevel methods (cf. $[12,13])$. By adapting Xu's proof of this result, we will prove the same for our decomposition and with that, convergence for the Laplace operator.

Our FDMLM can be seen as block Jacobi's method after a basis transformation to a certain hierarchical basis. Our convergence result means that independent of the dimension, the stiffness matrix after this transformation has a bounded condition number uniformly in the level and the anisotropy.

In a forthcoming paper ([11]), we will discuss an efficient implementation of the method and present numerical results.

Following [12], we shall use the notations $\lesssim, \gtrsim$ and $\underset{\bar{\sim}}{\sim}$. When we write

$$
x_{1} \lesssim y_{1}, x_{2} \gtrsim y_{2} \text { and } x_{3} \approx y_{3},
$$

there exist constants $C_{1}, c_{2}, c_{3}$ and $C_{3}$ independent of relevant parameters such as the level or the anisotropy, such that

$$
x_{1} \leq C_{1} y_{1}, x_{2} \geq c_{2} y_{2} \text { and } c_{3} x_{3} \leq y_{3} \leq C_{3} x_{3} .
$$




\section{Description OF THE METHOD}

2.1. Basic definitions. We start by giving some definitions for the one-dimensional case. Let $\Omega=(0,1), h_{k}=2^{-(k+1)}\left(k \in \mathbf{N}_{0}=\{0,1,2, \ldots\}\right)$ and let $\Omega_{k}^{2}=$ $\Omega \cap h_{k}\left(\mathbf{Z}+\frac{1}{2} \imath\right)(\imath \in\{0,1\})$. Note that $\Omega_{k}^{0}=\Omega_{k-1}^{0} \cup \Omega_{k-1}^{1}$ (cf. Figure 1$)$. We equip

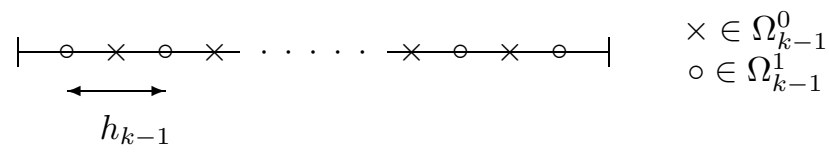

FiguRE 1. "Standard" and "shifted" coarse-grids $\Omega_{k-1}^{0}$ and $\Omega_{k-1}^{1}$ respectively

the space of grid functions on $\Omega_{k}^{2}$, denoted by $\ell^{2}\left(\Omega_{k}^{2}\right)$, with the Euclidean scalar product

$$
\langle\mu, \nu\rangle=\sum_{x \in \Omega_{k}^{2}} \mu(x) \overline{\nu(x)}
$$

and norm $\|\cdot\|=\langle\cdot, \cdot\rangle^{\frac{1}{2}}$.

The prolongations $p^{2}: \ell^{2}\left(\Omega_{k-1}^{2}\right) \rightarrow \ell^{2}\left(\Omega_{k}^{0}\right)$ are defined in difference stencil notation as $p^{0}=\frac{1}{2}$ ] $1 \quad 2 \quad 1$ [ (linear interpolation) and $p^{1}=\frac{1}{2}$ ] $-1 \quad 2 \quad-1$ [. They satisfy

$$
\text { range } p^{0} \oplus^{\perp} \text { range } p^{1}=\ell^{2}\left(\Omega_{k}^{0}\right) .
$$

The restrictions $r^{2}: \ell^{2}\left(\Omega_{k}^{0}\right) \rightarrow \ell^{2}\left(\Omega_{k-1}^{2}\right)$ are defined as adjoints of the corresponding prolongations, that is, $r^{0}=\frac{1}{2}\left[\begin{array}{lll}1 & 2 & 1\end{array}\right]$ and $r^{1}=\frac{1}{2}\left[\begin{array}{lll}-1 & 2 & -1\end{array}\right]$.

For the general d-dimensional case, we define the grids $\Omega_{k}^{\imath}=\Omega_{k_{1}}^{\imath_{1}} \times \cdots \times \Omega_{k_{d}}^{\imath_{d}}$ $\left(k \in \mathbf{N}_{0}^{d}, \imath \in\{0,1\}^{d}\right)$. We equip the space $\ell^{2}\left(\Omega_{k}^{\imath}\right)$ of grid functions on $\Omega_{k}^{\imath}$ also with the Euclidean scalar product $\langle\mu, \nu\rangle=\sum_{x \in \Omega_{k}^{2}} \mu(x) \overline{\nu(x)}$.

Since we exploit tensor products quite often, note that $\ell^{2}\left(\Omega_{k}^{2}\right)=\bigotimes_{j=1}^{d} \ell^{2}\left(\Omega_{k_{j}}^{\imath_{j}}\right)$, i.e., $\ell^{2}\left(\Omega_{k}^{2}\right)=\operatorname{span}\left\{\bigotimes_{j=1}^{d} \mu_{j}: \mu_{j} \in \ell^{2}\left(\Omega_{k_{j}}^{\imath_{j}}\right)\right\}$, where $\left(\bigotimes_{j=1}^{d} \mu_{j}\right)(x):=\prod_{j=1}^{d} \mu_{j}\left(x_{j}\right)$. Furthermore, we have $\left\langle\bigotimes_{j=1}^{d} \mu_{j}, \bigotimes_{j=1}^{d} \nu_{j}\right\rangle=\prod_{j=1}^{d}\left\langle\mu_{j}, \nu_{j}\right\rangle$.

2.2. Derivation of the (modified) FDMLM. First, we consider the two-dimensional case. In [2], the FD Two-Level Method to solve a system $\mathcal{A} \mu=\beta$ on $\Omega_{J J}^{00}$ was defined by

$$
\mu \leftarrow \mu-\sum_{\imath, \jmath \in\{0,1\}} p_{x}^{\imath} \otimes p_{y}^{\jmath}\left(r_{x}^{\imath} \otimes r_{y}^{\jmath} \mathcal{A} p_{x}^{\imath} \otimes p_{y}^{\jmath}\right)^{-1} r_{x}^{\imath} \otimes r_{y}^{\jmath}(\mathcal{A} \mu-\beta),
$$

where thus $r_{x}^{\imath} \otimes r_{y}^{\jmath} \mathcal{A} p_{x}^{\imath} \otimes p_{y}^{\jmath}$ acts on the space of grid functions on $\Omega_{J-1 J-1}^{\imath J}$ $\left(=\Omega_{J-1}^{2} \times \Omega_{J-1}^{J}\right)$. [It will be clear why we avoid the term two-grid method.] Using the abbreviations $p^{\imath \jmath}$ and $r^{\imath \jmath}$ for $p_{x}^{\imath} \otimes p_{y}^{\jmath}$ and $r_{x}^{\imath} \otimes r_{y}^{\jmath}$, respectively, we have

$$
p^{\imath \jmath}=\frac{1}{4}\left[\begin{array}{ccc}
(-1)^{\imath+\jmath} & (-1)^{\jmath} 2 & (-1)^{2+\jmath} \\
(-1)^{\imath 2} & 4 & (-1)^{\imath} 2 \\
(-1)^{\imath+\jmath} & (-1)^{\jmath} 2 & (-1)^{2+\jmath}
\end{array}[\right.
$$


and

$$
r^{\imath \jmath}\left(=\left(p^{\imath \jmath}\right)^{*}\right)=\frac{1}{4}\left[\begin{array}{ccc}
(-1)^{\imath+\jmath} & (-1)^{\jmath} 2 & (-1)^{\imath+\jmath} \\
(-1)^{\imath} 2 & 4 & (-1)^{\imath} 2 \\
(-1)^{\imath+\jmath} & (-1)^{\jmath} 2 & (-1)^{\imath+\jmath}
\end{array}\right] .
$$

We consider only $\mathcal{A}>0$. Then, because of the Galerkin approach, the error amplification operator of the method is given by

$$
\mu^{*}-\mu^{\text {new }}=\left(I-\sum_{\imath, \jmath \in\{0,1\}} \mathcal{P}^{\imath \jmath}\right)\left(\mu^{*}-\mu^{\text {old }}\right),
$$

where $\mu^{*}$ is the exact solution and $\mathcal{P}^{\imath \jmath}$ is the projection from $\ell^{2}\left(\Omega_{J J}^{00}\right)$ onto the range $p^{\imath \jmath}$ orthogonal with respect to $\langle\mathcal{A} \cdot, \cdot\rangle$. The range of the standard prolongation $p^{00}$ contains the "smooth" functions. The one-dimensional prolongation $p^{1}$ was chosen such that the ranges of the $p^{\imath \jmath}$ for $(\imath, \jmath) \neq(0,0)$ contain the different types of oscillating functions, so that also errors of that kind are corrected.

We consider systems that arise from the application of the bilinear finite element method to

$$
\left\{\begin{array}{rlll}
-\left(a_{1} \partial_{1}^{2}+a_{2} \partial_{2}^{2}\right) u & = & \text { on } \Omega^{2} \\
u & = & \text { on } \partial \Omega^{2}
\end{array}\right.
$$

that is,

$$
\mathcal{A}=\frac{1}{6} a_{1}\left[\begin{array}{lll}
-1 & 2 & -1
\end{array}\right]\left[\begin{array}{l}
1 \\
4 \\
1
\end{array}\right]+\frac{1}{6} a_{2}\left[\begin{array}{r}
-1 \\
2 \\
-1
\end{array}\right]\left[\begin{array}{lll}
1 & 4 & 1
\end{array}\right]: \ell^{2}\left(\Omega_{J J}^{00}\right) \rightarrow \ell^{2}\left(\Omega_{J J}^{00}\right),
$$

where $a_{1}, a_{2} \geq 0$ and $a_{1}+a_{2}>0$. This kind of problem is called anisotropic when $a_{1} \ll a_{2}$ or $a_{1} \gg a_{2}$. In [5], it was proved that the FDTLM yields a robust preconditioner, that is, the condition number $\kappa\left(\sum_{\imath, \jmath \in\{0,1\}} p^{\imath \jmath}\left(r^{\imath \jmath} \mathcal{A} p^{\imath \jmath}\right)^{-1} r^{\imath \jmath} \mathcal{A}\right) \lesssim 1$ (uniformly in $J$ and $a_{i}$ ). Our aim is to prove the same for a multilevel version.

In its original form, the multilevel version consisted of recursive calls for each of the four coarse-grid problems on the grids $\Omega_{J-1 J-1}^{00}, \Omega_{J-1 J-1}^{01}, \Omega_{J-1 J-1}^{10}$ and $\Omega_{J-1 J-1}^{11}$. By $r^{0}\left[\begin{array}{lll}-1 & 2 & -1\end{array}\right] p^{0}=\frac{1}{2}\left[\begin{array}{lll}-1 & 2 & -1\end{array}\right], r^{0}\left[\begin{array}{lll}1 & 4 & 1\end{array}\right] p^{0}=2\left[\begin{array}{lll}1 & 4 & 1\end{array}\right]$, $r^{1}\left[\begin{array}{lll}1 & 4 & 1\end{array}\right] p^{1}=4 I$ and

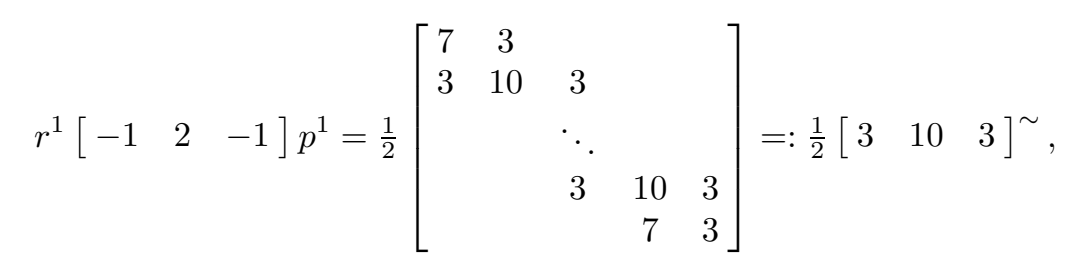

the operators on the spaces of grid functions on these grids are

$$
\begin{gathered}
r^{00} \mathcal{A} p^{00}=\frac{1}{6} a_{1}\left[\begin{array}{lll}
-1 & 2 & -1
\end{array}\right]\left[\begin{array}{l}
1 \\
4 \\
1
\end{array}\right]+\frac{1}{6} a_{2}\left[\begin{array}{r}
-1 \\
2 \\
-1
\end{array}\right]\left[\begin{array}{lll}
1 & 4 & 1
\end{array}\right] \quad \text { on } \ell^{2}\left(\Omega_{J-1 J-1}^{00}\right), \\
r^{01} \mathcal{A} p^{01}=\frac{1}{3} a_{1}\left[\begin{array}{lll}
-1 & 2 & -1
\end{array}\right]+\frac{1}{6} a_{2}\left[\begin{array}{r}
3 \\
10 \\
3
\end{array}\right]^{\sim}\left[\begin{array}{lll}
1 & 4 & 1
\end{array}\right] \quad \text { on } \ell^{2}\left(\Omega_{J-1 J-1}^{01}\right),
\end{gathered}
$$




$$
r^{10} \mathcal{A} p^{10}=\frac{1}{6} a_{1}\left[\begin{array}{lll}
3 & 10 & 3
\end{array}\right]^{\sim}\left[\begin{array}{l}
1 \\
4 \\
1
\end{array}\right]+\frac{1}{3} a_{2}\left[\begin{array}{r}
-1 \\
2 \\
-1
\end{array}\right] \quad \text { on } \ell^{2}\left(\Omega_{J-1 J-1}^{10}\right)
$$

and

$$
r^{11} \mathcal{A} p^{11}=\frac{1}{3} a_{1}\left[\begin{array}{lll}
3 & 10 & 3
\end{array}\right]^{\sim}+\frac{1}{3} a_{2}\left[\begin{array}{r}
3 \\
10 \\
3
\end{array}\right]^{\sim} \quad \text { on } \ell^{2}\left(\Omega_{J-1 J-1}^{11}\right) .
$$

As noted in [3], there holds $\kappa\left(r^{11} \mathcal{A} p^{11}\right) \lesssim 1$ (uniformly in the grid sizes and $a_{i}$ ). The argument is that for $\mathcal{B}_{1}, \mathcal{B}_{2}>0$, we have $\kappa\left(\mathcal{B}_{1}+\mathcal{B}_{2}\right) \leq \max \left\{\kappa\left(\mathcal{B}_{1}\right), \kappa\left(\mathcal{B}_{2}\right)\right\}$. So instead of applying a recursive call, the corresponding system can be solved using a cheap iterative solver. Furthermore, in [3] it was argued that in the cases $a_{1} \leq a_{2}$ or $a_{1} \geq a_{2}$ also one of the two operators $r^{01} \mathcal{A} p^{01}$ and $r^{10} \mathcal{A} p^{10}$ has a bounded condition number. Yet, this argument cannot be applied to construct a method that is robust for the general variable-coefficient case. Therefore, we will use another idea to further reduce the number of recursive calls.

Consider the following two operators that arise from $\tilde{\mathcal{A}}:=r^{01} \mathcal{A} p^{01}$ by means of semicoarsening $\Omega_{J-1 J-1}^{01}$ in the $x$-direction, that is, in the direction where we have not applied $p^{1}$ so far,

$$
r_{x}^{0} \tilde{\mathcal{A}} p_{x}^{0}=\frac{1}{6} a_{1}\left[\begin{array}{lll}
-1 & 2 & -1
\end{array}\right]+\frac{1}{3} a_{2}\left[\begin{array}{r}
3 \\
10 \\
3
\end{array}\right]^{\sim}\left[\begin{array}{lll}
1 & 4 & 1
\end{array}\right] \quad \text { on } \ell^{2}\left(\Omega_{J-2 J-1}^{01}\right)
$$

and

$$
r_{x}^{1} \tilde{\mathcal{A}} p_{x}^{1}=\frac{1}{6} a_{1}\left[\begin{array}{lll}
3 & 10 & 3
\end{array}\right]^{\sim}+\frac{2}{3} a_{2}\left[\begin{array}{r}
3 \\
10 \\
3
\end{array}\right]^{\sim} \quad \text { on } \ell^{2}\left(\Omega_{J-2 J-1}^{11}\right) .
$$

Then the first operator is of the same type as $\tilde{\mathcal{A}}$, and so we can apply $(x$-)semicoarsening recursively or, if $J-2=0$, the operator has a bounded condition number and therefore the system can be solved using a cheap iterative solver. The second operator always has a bounded condition number.

Analogously to the above procedure, we can solve the system on $\Omega_{J-1 J-1}^{10}$ using semicoarsening in the $y$-direction. Finally, as with the original version, the system on $\Omega_{J-1 J-1}^{00}$ is solved with a recursive call of the entire method, with which this informal description of the modified FDMLM is completed (see Figure 2).

In view of the following, note that since, e.g., on $\Omega_{J-1 J-1}^{01}$ no system is solved (unless $J-1=0$ ), but only coarse-grid corrections are invoked, this (intermediate) grid and the operators defined on it are only important for an efficient implementation. Because of the Galerkin approach, the mathematical properties of the resulting method are determined by the (sequence of) prolongations from the grids on which systems are (approximately) solved (leaves in the tree of Figure 2) onto the finest grid $\Omega_{J J}^{00}$. For example, for $J=2$ these prolongations are $p_{x}^{0} p_{x}^{0} \otimes p_{y}^{0} p_{y}^{0}$, $p_{x}^{0} p_{x}^{0} \otimes p_{y}^{0} p_{y}^{1}, p_{x}^{0} p_{x}^{0} \otimes p_{y}^{1}, p_{x}^{0} p_{x}^{1} \otimes p_{y}^{0} p_{y}^{0}, p_{x}^{0} p_{x}^{1} \otimes p_{y}^{0} p_{y}^{1}, p_{x}^{0} p_{x}^{1} \otimes p_{y}^{1}, p_{x}^{1} \otimes p_{y}^{0} p_{y}^{0}, p_{x}^{1} \otimes p_{y}^{0} p_{y}^{1}$ and $p_{x}^{1} \otimes p_{y}^{1}$, i.e., tensor products of all possible combinations of $p_{x}^{0} p_{x}^{0}, p_{x}^{0} p_{x}^{1}, p_{x}^{1}$ and $p_{y}^{0} p_{y}^{0}, p_{y}^{0} p_{y}^{1}, p_{y}^{1}$.

We are now ready to give a formal description of the modified FDMLM. 


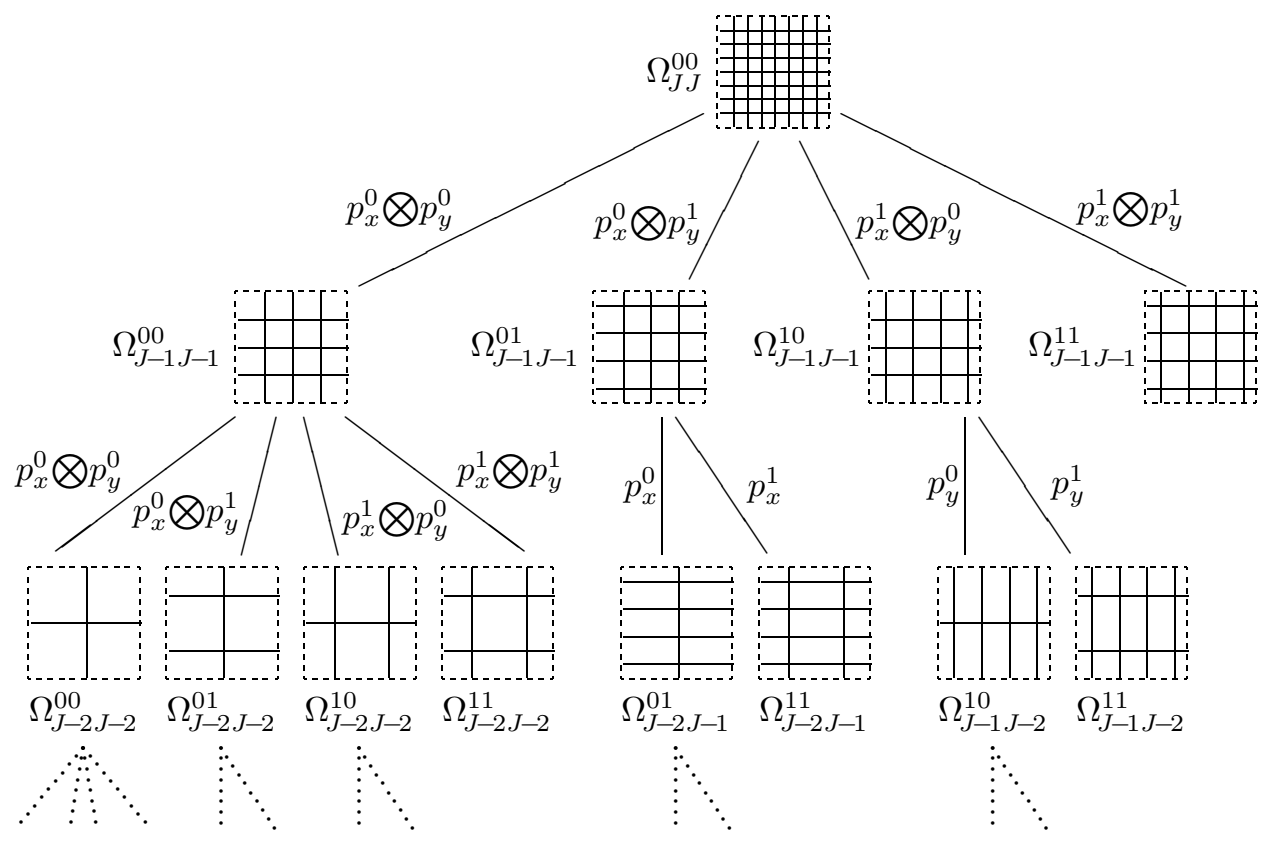

Figure 2. Grids and prolongations defining the modified FDMLM in two dimensions. [ We dotted the lines at the bottom of the figure since the pictures of the grids correspond to $J=2$, i.e., the three-level case.]

Algorithm 2.1. Let $\mathcal{A} \mu=\beta$ be a system on the $d$-dimensional grid $\Omega_{J \cdots J}^{0 \cdots 0}$. For $0 \leq k \leq J$, define the one-dimensional prolongation $p_{k}=p_{k}^{(J)}$ by

$$
p_{k}=\left\{\begin{array}{lll}
\underbrace{p^{0} \cdots p^{0} p^{1}}_{(J-k) \times} & : \ell^{2}\left(\Omega_{k-1}^{1}\right) \rightarrow \ell^{2}\left(\Omega_{J}^{0}\right), & k \geq 1, \\
\underbrace{p^{0} \cdots p^{0}}_{J \times} & : \ell^{2}\left(\Omega_{0}^{0}\right) \rightarrow \ell^{2}\left(\Omega_{J}^{0}\right), & k=0 .
\end{array}\right.
$$

Note that from (1) we have $\ell^{2}\left(\Omega_{J}^{0}\right)=\bigoplus_{k=0}^{J}$ range $p_{k}$. For $k \in I:=\{0, \ldots, J\}^{d}$, we define

$$
p_{k}=\bigotimes_{j=1}^{d} p_{k_{j}, x_{j}}, \quad r_{k}=p_{k}^{*}\left(=\bigotimes_{j=1}^{d} r_{k_{d}, x_{d}}\right) \quad \text { and } \mathcal{A}_{k}=r_{k} \mathcal{A} p_{k} .
$$

Now let $\mathcal{B}_{k}$ be such that $\mathcal{B}_{k}^{-1}$ is a cheap approximation of $\mathcal{A}_{k}^{-1}$. Then the (modified) FDMLM is defined by

$$
\mu \leftarrow \mu-\sum_{k \in I} p_{k} \mathcal{B}_{k}^{-1} r_{k}(\mathcal{A} \mu-\beta) .
$$

Our FDMLM is an example of an additive Schwarz method or Parallel Subspace Correction method with subspace range $p_{k}$ satisfying $\ell^{2}\left(\Omega_{J \cdots J}^{0 \cdots 0}\right)=\bigoplus_{k \in I}$ range $p_{k}$.

Remark 2.2. Since, e.g., $\Omega_{J-1 J-1}^{01}(d=2)$ is coarsened only in the $x$-direction, the elementary one-dimensional prolongations $p^{0}$ and $p^{1}$, which are the building blocks 
of all prolongations in the algorithm, always map onto the space of grid functions on a non-shifted grid, that is, a grid $\Omega_{k}^{0}$ for some $k \in \mathbf{N}$. So in contrast to the original FDMLM, we do not have to construct boundary adaptations for $p^{0}$ and $p^{1}$ in order to maintain property (1).

We want to prove robustness of this method applied to anisotropic problems. As a consequence of the following lemma it is then sufficient to analyze the FDMLM with exact subspace corrections $\left(\mathcal{B}_{k}=\mathcal{A}_{k}\right)$. The straightforward proof of this lemma is left to the reader.

Lemma 2.3. Let $\mathcal{A}>0$ and $\mathcal{B}_{k}>0(k \in I)$. Define $\Lambda=\max _{k \in I} \lambda_{\max }\left(\mathcal{B}_{k}^{-1} \mathcal{A}_{k}\right)$ and $\lambda=\min _{k \in I} \lambda_{\min }\left(\mathcal{B}_{k}^{-1} \mathcal{A}_{k}\right)$. Then

$$
\kappa\left(\sum_{k \in I} p_{k} \mathcal{B}_{k}^{-1} r_{k} \mathcal{A}\right) \leq \frac{\Lambda}{\lambda} \kappa\left(\sum_{k \in I} p_{k} \mathcal{A}_{k}^{-1} r_{k} \mathcal{A}\right) .
$$

Analogously to the two-dimensional case, for $d$-dimensional anisotropic problems we have that $\kappa\left(\mathcal{A}_{k}\right) \lesssim 1(k \in I)$. So already the simple Richardson iteration, that is $\mathcal{B}_{k}=\rho\left(\mathcal{A}_{k}\right)$, gives $\frac{\Lambda}{\lambda}=\max _{k \in I} \kappa\left(\mathcal{A}_{k}\right) \lesssim 1$.

2.3. Computational complexity. For ease of presentation we consider the twodimensional case. The general case can be handled by induction.

Assume that the application of $\mathcal{B}_{k}^{-1}(k \in I)$ costs a number of operations that is equivalent to the number of points of the grid in question. For $k_{1} \leq k_{2}$, let $W_{k_{1} k_{2}}^{01}\left(W_{k_{2} k_{1}}^{10}\right)$ be the number of arithmetic operations necessary to treat a system on $\Omega_{k_{1} k_{2}}^{01}\left(\Omega_{k_{2} k_{1}}^{10}\right)$ using the recursive application of semicoarsening in the $x$ - $(y$ - $)$ direction. Then we have $W_{k_{1} k_{2}}^{01} \bar{\sim} \# \Omega_{k_{1} k_{2}}^{01}+W_{k_{1}-1 k_{2}}^{01}$, which gives $W_{k_{1} k_{2}}^{01} \equiv \# \Omega_{k_{1} k_{2}}^{01}$ and analogously $W_{k_{2} k_{1}}^{10} \approx \# \Omega_{k_{2} k_{1}}^{10}$. Finally, let $W_{k k}^{00}$ be the number of arithmetic operations necessary for an entire FDMLM call on $\Omega_{k k}^{00}$. We conclude that

$$
\begin{aligned}
W_{k k}^{00} & \equiv W_{k-1 k-1}^{00}+W_{k-1 k-1}^{01}+W_{k-1 k-1}^{10}+\# \Omega_{k k}^{00} \\
& \equiv W_{k-1 k-1}^{00}+\# \Omega_{k k}^{00},
\end{aligned}
$$

which implies $W_{k k}^{00} \approx \# \Omega_{k k}^{00}$. Note that since $\# \Omega_{k-1 k-1}^{00} / \# \Omega_{k k}^{00}=\frac{1}{4}$, more than one recursive call on $\Omega_{k-1 k-1}^{00}$ can be applied. The number of recursive calls involving semicoarsening should be restricted to one.

\section{Proof of Robustness of the FDMLM}

3.1. Coordinate-free finite element formulation. To facilitate the analysis, we reformulate the algorithm in a more abstract context. We start by giving some definitions for the one-dimensional case.

For $\imath \in\{0,1\}, k \in \mathbf{N}_{0}, x \in \Omega_{k}^{2}$, define $\delta_{k, x}^{\imath} \in \ell^{2}\left(\Omega_{k}^{2}\right)$ by $\delta_{k, x}^{\imath}(y)= \begin{cases}1, & x=y, \\ 0, & x \neq y .\end{cases}$

Define $P_{k}: \ell^{2}\left(\Omega_{k}^{0}\right) \rightarrow H_{0}^{1}(\Omega) \subset L^{2}(\Omega)$ as the linear interpolation operator using zero boundary values. Put $\mathcal{M}_{k}=$ range $P_{k}$, that is, $\mathcal{M}_{k}$ is the linear finite element space corresponding to the grid $\Omega_{k}^{0}$. The basis $\left\{\phi_{k, x}^{0}:=P_{k} \delta_{k, x}^{0}: x \in \Omega_{k}^{0}\right\}$ is the standard (nodal) basis of $\mathcal{M}_{k}$. We equip $\mathcal{M}_{k}$ with scalar product

$$
\langle\cdot, \cdot\rangle_{\mathcal{M}_{k}}=h_{k}\left\langle P_{k}^{-1} \cdot, P_{k}^{-1} \cdot\right\rangle
$$

and norm $\|\cdot\|_{\mathcal{M}_{k}}=\langle\cdot, \cdot\rangle_{\mathcal{M}_{k}}^{\frac{1}{2}}$. It is well known that $\|\cdot\|_{\mathcal{M}_{k}} \approx\|\cdot\|_{L^{2}}$ (uniformly in $k)$. 
For $k>1$, we define $\mathcal{V}_{k}=\operatorname{range}\left(P_{k} p^{1}: \ell^{2}\left(\Omega_{k-1}^{1}\right) \rightarrow H_{0}^{1}(\Omega)\right)$. We will call the basis $\left\{\phi_{k, x}^{1}:=P_{k} p^{1} \delta_{k-1, x}^{1}: x \in \Omega_{k-1}^{1}\right\}$ the standard basis of $\mathcal{V}_{k}$. Using $P_{k} p^{0}=P_{k-1}$, we find that (1) is equivalent to

$$
\mathcal{M}_{k}=\mathcal{M}_{k-1} \oplus^{\perp \mathcal{M}_{k}} \mathcal{V}_{k}
$$

So, with the definition $\mathcal{V}_{0}=\mathcal{M}_{0}$, the union of the bases of $\mathcal{V}_{0}, \ldots, \mathcal{V}_{k}$ forms a basis of $\mathcal{M}_{k}$, which is therefore called a hierarchical basis (cf. Figure 3). Note that (4)

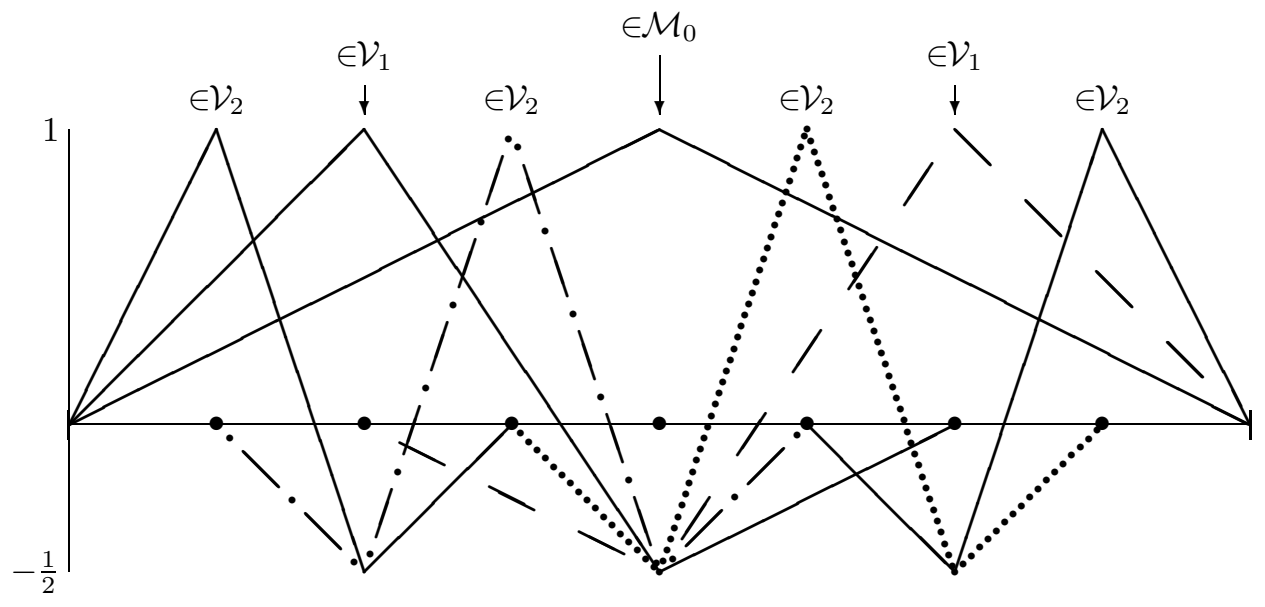

FIgURE 3. Hierarchical basis of $\mathcal{M}_{2}$

does not imply that $\mathcal{V}_{0}, \ldots, \mathcal{V}_{k}$ are mutually orthogonal with respect to some scalar product.

For $k \in \mathbf{N}_{0}$, let $I_{k}: \mathcal{V}_{k} \rightarrow L^{2}(\Omega)$ be the inclusion operator. Since

$$
P_{J} p_{k}=\left\{\begin{array}{cl}
P_{J} \underbrace{p^{0} \cdots p^{0}}_{(J-k) \times} p^{1}=P_{k} p^{1}, & 1 \leq k \leq J, \\
P_{J} \underbrace{p^{0} \cdots p^{0}}_{J \times}=P_{0}, & k=0,
\end{array}\right.
$$

we find that $p_{k}$ is the representation of $I_{k}$ with respect to the standard bases on $\mathcal{V}_{k}$ and $\mathcal{M}_{J}$.

As usual, for some basis $\left\{\psi_{i}: i \in I_{\mathcal{W}}\right\}$ of a subspace $\mathcal{W} \subset L^{2}(\Omega)$, we define the dual basis $\left\{\tilde{\psi}_{i}: i \in I_{\mathcal{W}}\right\}$ of $\mathcal{W}$ by $\left(\tilde{\psi}_{i}, \psi_{j}\right)_{L^{2}}=\left\{\begin{array}{ll}1, & i=j, \\ 0, & i \neq j .\end{array} \quad\right.$ Let $V_{k}: L^{2}(\Omega) \rightarrow \mathcal{V}_{k}$ be the adjoint of $I_{k}$ with respect to the $L^{2}$-scalar product on both spaces, that is, $V_{k}$ is the $L^{2}$-orthogonal projection onto $\mathcal{V}_{k}$. Then we find that the representation of $\left.V_{k}\right|_{\mathcal{M}_{J}}$ with respect to the dual bases on $\mathcal{M}_{J}$ and $\mathcal{V}_{k}$ is equal to the adjoint of $p_{k}$, that is, $r_{k}$. is,

In the multidimensional case everything is defined using tensor products, that

$$
\mathcal{M}_{k}=\bigotimes_{j=1}^{d} \mathcal{M}_{k_{j}}, \quad \mathcal{V}_{k}=\bigotimes_{j=1}^{d} \mathcal{V}_{k_{j}}, \quad I_{k}=\bigotimes_{j=1}^{d} I_{k_{j}} \text { and } V_{k}\left(=I_{k}^{*}\right)=\bigotimes_{j=1}^{d} V_{k_{j}}
$$


So, $I_{k}: \mathcal{V}_{k} \rightarrow L^{2}\left(\Omega^{d}\right)$ is the inclusion operator and $V_{k}: L^{2}\left(\Omega^{d}\right) \rightarrow \mathcal{V}_{k}$ is the $L^{2}$ orthogonal projection onto $\mathcal{V}_{k}$. We equip $\mathcal{M}_{k}$ and $\mathcal{V}_{k}$ with standard bases obtained by making tensor products of the standard basis functions of its factors, that is, the standard bases consist of functions of the form $\phi_{k, x}^{2}=\bigotimes_{j=1}^{d} \phi_{k_{j}, x_{j}}^{i_{j}}$. Concerning dual basis functions, note that $\tilde{\phi}_{k, x}^{2}=\bigotimes_{j=1}^{d} \tilde{\phi}_{k_{j}, x_{j}}^{r_{j}}$. Using the abbreviation $\mathbf{m}$ for multi-indices $(m, \ldots, m) \in \mathbf{N}_{0}^{d}$, we conclude that for $k \in I, p_{k}$ is the representation of $I_{k}$ with respect to the standard bases on $\mathcal{V}_{k}$ and $\mathcal{M}_{\mathbf{J}}$, and $r_{k}$ is the representation of $\left.V_{k}\right|_{\mathcal{M}_{\mathrm{J}}}$ with respect to the dual bases.

Finally, for a given system $\mathcal{A} \mu=\beta$ on $\Omega_{\mathbf{J}}^{0}$, define $A: \mathcal{M}_{\mathbf{J}} \rightarrow \mathcal{M}_{\mathbf{J}}$ by

$$
\left(A \phi_{\mathbf{J}, y}^{\mathbf{0}}, \phi_{\mathbf{J}, x}^{\mathbf{0}}\right)_{L^{2}}=\mathcal{A}_{x y} \quad\left(x, y \in \Omega_{\mathbf{J}}^{\mathbf{0}}\right) .
$$

Then $\mathcal{A}$ is the representation of $A$ with respect to the standard basis on its domain and dual basis on its image. With the definition $A_{k}=V_{k} A I_{k}$, we arrive at the conclusion that (2), with $\mathcal{B}_{k}=\mathcal{A}_{k}$, is a matrix formulation of the iteration

$$
u \leftarrow u-\sum_{k \in I} I_{k} A_{k}^{-1} V_{k}(A u-f),
$$

where $u=\sum_{x \in \Omega_{\mathbf{J}}^{\mathbf{o}}} \mu(x) \phi_{\mathbf{J}, x}^{\mathbf{0}}, f=\sum_{x \in \Omega_{\mathbf{J}}^{\mathbf{0}}} \beta(x) \tilde{\phi}_{\mathbf{J}, x}^{\mathbf{0}}$ (that is, $\left.\beta(x)=\left(f, \phi_{\mathbf{J}, x}^{\mathbf{0}}\right)_{L^{2}}\right)$. Because the condition number $\kappa$ was defined as the quotient of the largest and smallest eigenvalue, clearly we have $\kappa\left(\sum_{k \in I} p_{k} \mathcal{A}_{k}^{-1} r_{k} \mathcal{A}\right)=\kappa\left(\sum_{k \in I} I_{k} A_{k}^{-1} V_{k} A\right)$.

Remark 3.1. We defined the operator $A$ using the matrix $\mathcal{A}$. Of course, the usual procedure is the other way around. If $a$ is a bilinear form on $H_{0}^{1}\left(\Omega^{d}\right)$ and $A: \mathcal{M}_{\mathbf{J}} \rightarrow$ $\mathcal{M}_{\mathbf{J}}$ is defined by $(A u, v)_{L^{2}}=a(u, v)\left(u, v \in \mathcal{M}_{J}\right)$, then $\mathcal{A}$ defined by (5) is called the stiffness matrix with respect to the (multilinear) basis $\left\{\phi_{\mathbf{J}, x}^{\mathbf{0}}: x \in \Omega_{\mathbf{J}}^{\mathbf{0}}\right\}$.

Remark 3.2. Consider the hierarchical basis of $\mathcal{M}_{\mathbf{J}}=\bigoplus_{k \in I} \mathcal{V}_{k}$ that is obtained by taking the union of the standard bases of the $\mathcal{V}_{k}$. The iteration (6) with respect to this basis, that is, the hierarchical basis for the solution and its dual for the right-hand side, is just block Jacobi's method with a partitioning into blocks corresponding to the spaces $\mathcal{V}_{k}$. As we have seen, for anisotropic problems, the diagonal blocks have bounded condition number and so robustness of (6) implies that, properly scaled, the stiffness matrix with respect to this hierarchical basis has a bounded condition number uniformly in the level and the anisotropy.

3.2. Main theorem; reduction to one-dimensional cases. The fact that $\mathcal{M}_{\mathbf{J}}=\bigoplus_{k \in I} \mathcal{V}_{k}$ is a direct sum decomposition implies that there exist projections $Z_{k}=Z_{k}^{(\mathbf{J})}: \mathcal{M}_{\mathbf{J}} \rightarrow \mathcal{V}_{k}$ such that $\sum_{k \in I} Z_{k}=I$ on $\mathcal{M}_{\mathbf{J}}$. Note that $Z_{k} I_{k^{\prime}}=0$ if $k \neq k^{\prime}$ and that $Z_{k} I_{k}$ is the identity on $\mathcal{V}_{k}$.

Lemma 3.3. Define $W=\sum_{k \in I} Z_{k}^{*} A_{k} Z_{k}: \mathcal{M}_{\mathbf{J}} \rightarrow \mathcal{M}_{\mathbf{J}}$. Then $W^{-1}$ exists and is equal to $\sum_{k \in I} I_{k} A_{k}^{-1} V_{k}$.

Proof. $\sum_{k \in I} Z_{k}^{*} A_{k} Z_{k} \sum_{k^{\prime} \in I} I_{k^{\prime}} A_{k^{\prime}}^{-1} V_{k^{\prime}}=\sum_{k \in I} Z_{k}^{*} V_{k}=\left(\sum_{k \in I} I_{k} Z_{k}\right)^{*}=I$.

This lemma shows that for $A>0$, the condition number $\kappa\left(\sum_{k \in I} I_{k} A_{k}^{-1} V_{k} A\right)$ is the quotient $\frac{\Gamma}{\gamma}$ of the optimum constants in the inequalities $\gamma W \leq A \leq \Gamma W$ or, by $A_{k}=V_{k} A I_{k}$,

$$
\gamma \sum_{k \in I}\left(A Z_{k} u, Z_{k} u\right)_{L^{2}} \leq(A u, u)_{L^{2}} \leq \Gamma \sum_{k \in I}\left(A Z_{k} u, Z_{k} u\right)_{L^{2}} \quad\left(u \in \mathcal{M}_{\mathbf{J}}\right) .
$$


We are now ready to formulate our main theorem.

Theorem 3.4. For nonnegative constants $a_{j}$ and $b$ with $\sum_{j=1}^{d} a_{j}+b>0$, let

$$
a(u, v)=\sum_{j=1}^{d} \int_{\Omega^{d}} a_{j} \partial_{j} u \overline{\partial_{j} v}+\int_{\Omega^{d}} b u \bar{v} \quad\left(u, v \in H_{0}^{1}\left(\Omega^{d}\right)\right)
$$

and let $A: \mathcal{M}_{\mathbf{J}} \rightarrow \mathcal{M}_{\mathbf{J}}$ be defined by $(A u, v)_{L^{2}}=a(u, v)\left(u, v \in \mathcal{M}_{\mathbf{J}}\right)$. Then we have $\kappa\left(\sum_{k \in I} I_{k} A_{k}^{-1} V_{k} A\right) \lesssim 1$ (uniformly in $J, a_{j}$ and $b$ ).

Remark 3.5. From (7), we immediately see that the theorem can be extended to all operators $\tilde{A}$ for which there exist $c, C>0$ such that

$$
c(A u, u)_{L^{2}} \leq(\tilde{A} u, u)_{L^{2}} \leq C(A u, u)_{L^{2}}
$$

for some $A$ as described in the theorem. Examples are linear finite element discretizations or discretizations of elliptic boundary value problems with nonconstant coefficients. With a view to the nonconstant-coefficient case, we note that clearly the theorem does not yield boundedness of the condition number that is uniform in $C / c$.

To prove Theorem 3.4, we first note that if (7) is satisfied by $A^{(1)}$ and $A^{(2)}$, then it is satisfied by $c_{1} A^{(1)}+c_{2} A^{(2)}$ for any $c_{1}, c_{2} \geq 0$. As a consequence, we only have to consider tensor product operators $A=\bigotimes_{j=1}^{d} A_{j}$, where $\left(A_{j} u, u\right)_{L^{2}}=(u, u)_{L^{2}}$ or $\left(A_{j} u, u\right)_{L^{2}}=\left(u^{\prime}, u^{\prime}\right)_{L^{2}}\left(u \in \mathcal{M}_{J}\right)$. Secondly, we observe that (7) is equivalent to

$$
\sigma\left(\sum_{k \in I} A^{-\frac{1}{2}} Z_{k}^{*} A Z_{k} A^{-\frac{1}{2}}\right) \subset\left[\frac{1}{\Gamma}, \frac{1}{\gamma}\right] .
$$

As a special case of the general definition, the one-dimensional $Z_{k}^{(J)}: \mathcal{M}_{J} \rightarrow \mathcal{V}_{k}$ were defined by $\sum_{k=0}^{J} Z_{k}^{(J)}=I$. For $k \in I$, we have $Z_{k}\left(=Z_{k}^{(\mathbf{J})}\right)=\bigotimes_{j=1}^{d} Z_{k_{j}}^{(J)}$. From

$$
\begin{aligned}
& \sigma\left(\sum_{k \in I} A^{-\frac{1}{2}} Z_{k}^{*} A Z_{k} A^{-\frac{1}{2}}\right)=\sigma\left(\sum_{k \in I} \bigotimes_{j=1}^{d} A_{j}^{-\frac{1}{2}} Z_{k_{j}}^{(J)^{*}} A_{j} Z_{k_{j}}^{(J)} A_{j}^{-\frac{1}{2}}\right) \\
& =\sigma\left(\bigotimes_{j=1}^{d} \sum_{k=0}^{J} A_{j}^{-\frac{1}{2}} Z_{k_{j}}^{(J)^{*}} A_{j} Z_{k_{j}}^{(J)} A_{j}^{-\frac{1}{2}}\right)=\prod_{j=1}^{d} \sigma\left(\sum_{k=0}^{J} A_{j}^{-\frac{1}{2}} Z_{k_{j}}^{(J)^{*}} A_{j} Z_{k_{j}}^{(J)} A_{j}^{-\frac{1}{2}}\right),
\end{aligned}
$$

and again by using the equivalence of (8) and (7) but now for the one-dimensional case, we conclude that it suffices to prove the following norm equivalences in one dimension:

$$
\sum_{k=0}^{J}\left\|Z_{k}^{(J)} u\right\|_{L^{2}}^{2} \equiv\|u\|_{L^{2}}^{2}
$$

and

$$
\sum_{k=0}^{J}\left\|Z_{k}^{(J)} u\right\|_{H^{1}}^{2}=\|u\|_{H^{1}}^{2}
$$

$\left(u \in \mathcal{M}_{J}\right)$, where $\|\cdot\|_{H^{1}}:=(\cdot, \cdot)_{H^{1}}^{\frac{1}{2}}$ and $(u, v)_{H^{1}}:=\left(u^{\prime}, v^{\prime}\right)_{L^{2}}$.

We start with constructing an explicit formula for the one-dimensional projections $Z_{k}^{(J)}$ : 
Lemma 3.6. Let $Y_{k}: \mathcal{M}_{k+1} \rightarrow \mathcal{M}_{k}$ be the projection on $\mathcal{M}_{k}$ orthogonal with respect to $\langle\cdot, \cdot\rangle_{\mathcal{M}_{k+1}}$. Then

$$
Z_{k}^{(J)}= \begin{cases}Y_{0} \cdots Y_{J-1}, & k=0 \\ \left(I-Y_{k-1}\right) Y_{k} \cdots Y_{J-1}, & 1 \leq k \leq J\end{cases}
$$

Proof. From $\mathcal{M}_{J-1} \oplus^{\perp \mathcal{M}_{J}} \mathcal{V}_{J}=M_{J}$ and $M_{J-1}=\bigoplus_{k=1}^{J-1} \mathcal{V}_{k}$, it follows that $Z_{J}^{(J)}=$ $\left(I-Y_{J-1}\right)$ and $Z_{k}^{(J)}=Z_{k}^{(J-1)} Y_{J-1}(0 \leq k \leq J-1)$.

Remark 3.7. As noted before, $\|\cdot\|_{\mathcal{M}_{k}} \equiv\|\cdot\|_{L^{2}}$ on $\mathcal{M}_{k}$ (uniformly in $k$ ). If $\|\cdot\|_{\mathcal{M}_{k}}$ would be equal to $\|\cdot\|_{L^{2}}$ (which is not the case), then $Z_{k}^{(J)}$ defined by (11) would be equal to $\left.\left(Q_{k}-Q_{k-1}\right)\right|_{\mathcal{M}_{J}}$, where $Q_{k}: L^{2}(\Omega) \rightarrow \mathcal{M}_{k}$ is the $L^{2}$-orthogonal projection onto $\mathcal{M}_{k}$ and $Q_{-1}:=0$. For the decomposition $u=\sum_{k=0}^{J}\left(Q_{k}-Q_{k-1}\right) u$, (9) is trivially true. The corresponding relation (10) is famous and is the key to the modern regularity-free convergence proofs of standard multilevel methods (cf. $[12,13])$. Unlike the decomposition $u=\sum_{k=0}^{J}\left(Q_{k}-Q_{k-1}\right) u$, our decomposition was not introduced as a clever trick for the analysis of an overlapping subspace correction method, but it was yielded by the method itself.

In the next two subsections, $\S \S 3.3$ and 3.4 , we will prove the norm equivalences (9) and (10), respectively. We will prove (9) by estimating the angles between the spaces $\mathcal{V}_{k}$ with respect to the $L^{2}$-scalar product for our one-dimensional regular grid case. For the same case, an alternative proof exploiting the standard bases of the $\mathcal{V}_{k}$ appeared in [7]. We will prove (10) in an abstract framework using (9), $\sum_{k=0}^{J}\left\|\left(Q_{k}-Q_{k-1}\right) u\right\|_{H^{1}}^{2} \equiv\|u\|_{H^{1}}^{2}\left(u \in \mathcal{M}_{J}\right)$ and $\|\cdot\|_{\mathcal{M}_{k}} \equiv\|\cdot\|_{L^{2}}$ on $\mathcal{M}_{k}$.

3.3. An $L^{2}$-equivalent norm on $\mathcal{M}_{J}$. Since $Z_{k}^{(J)}$ is the projection from $\mathcal{M}_{J}$ onto $\mathcal{V}_{k}$ satisfying $\sum_{k=0}^{J} Z_{k}^{(J)}=I$, the validity of (9) depends on the angle between the spaces $\mathcal{V}_{k}$.

Lemma 3.8. Let $\theta_{k l}$ be the smallest constant satisfying

$$
\left|(u, v)_{L^{2}}\right| \leq \theta_{k l}\|u\|_{L^{2}}\|v\|_{L^{2}} \quad \text { for all } u \in \mathcal{V}_{k}, v \in \mathcal{V}_{l}
$$

(strengthened Cauchy-Schwarz inequality). Put $\Theta=\left(\theta_{k l}\right)_{k, l}$ and let $\rho(\Theta-I)<1$. Then

$$
1-\rho(\Theta-I) \leq\|u\|_{L^{2}}^{2} / \sum_{k=0}^{J}\left\|Z_{k}^{(J)} u\right\|_{L^{2}}^{2} \leq 1+\rho(\Theta-I) \quad\left(u \in \mathcal{M}_{J}\right) .
$$

Proof. Use

$$
\|u\|_{L^{2}}^{2}=\left(\sum_{k=0}^{J} Z_{k}^{(J)} u, \sum_{l=0}^{J} Z_{l}^{(J)} u\right)_{L^{2}}=\sum_{k=0}^{J}\left\|Z_{k}^{(J)} u\right\|_{L^{2}}^{2}+\sum_{0 \leq k \neq l \leq J}\left(Z_{k}^{(J)} u, Z_{l}^{(J)} u\right)_{L^{2}}
$$

and

$$
\begin{aligned}
\left|\sum_{0 \leq k \neq l \leq J}\left(Z_{k}^{(J)} u, Z_{l}^{(J)} u\right)_{L^{2}}\right| & \leq \sum_{0 \leq k \neq l \leq J} \theta_{k l}\left\|Z_{k}^{(J)} u\right\|_{L^{2}}\left\|Z_{l}^{(J)} u\right\|_{L^{2}} \\
& \leq \rho(\Theta-I) \sum_{k=0}^{J}\left\|Z_{k}^{(J)} u\right\|_{L^{2}}^{2} . \square
\end{aligned}
$$


Proposition 3.9. For $k>l$, there holds

$$
\theta_{k l} \leq\|\left(r^{1} M_{k} p^{1}\right)^{-\frac{1}{2}} r^{1} M_{k} \underbrace{p^{0} \cdots p^{0}}_{(k-l) \times} M_{l}^{-\frac{1}{2}}\|,
$$

where $M_{m}:=R_{m} P_{m}$ (mass matrix) and $R_{m}$ is the adjoint of $P_{m}: \ell^{2}\left(\Omega_{m}^{0}\right) \rightarrow L^{2}(\Omega)$.

Proof. The result follows from

$$
\begin{aligned}
& \theta_{k l}=\sup _{\substack{0 \neq u \in \mathcal{V}_{k} \\
0 \neq v \in \mathcal{V}_{l}}} \frac{\left|(u, v)_{L^{2}}\right|}{\|u\|_{L^{2}}\|v\|_{L^{2}}} \leq \sup _{\substack{0 \neq u \in \mathcal{V}_{k} \\
0 \neq v \in \mathcal{M}_{l}}} \frac{\left|(u, v)_{L^{2}}\right|}{\|u\|_{L^{2}}\|v\|_{L^{2}}} \\
& =\sup _{\substack{0 \neq \mu \in \ell^{2}\left(\Omega_{k-1}^{1}\right) \\
0 \neq \nu \in \ell^{2}\left(\Omega_{l}^{0}\right)}} \frac{\left|\left(P_{k} p^{1} \mu, P_{l} \nu\right)_{L^{2}}\right|}{\left(P_{k} p^{1} \mu, P_{k} p^{1} \mu\right)_{L^{2}}^{\frac{1}{2}}\left(P_{l} \nu, P_{l} \nu\right)_{L^{2}}^{\frac{1}{2}}} \\
& =\sup _{\substack{0 \neq \mu \in \ell^{2}\left(\Omega_{k-1}^{1}\right) \\
0 \neq \nu \in \ell^{2}\left(\Omega_{l}^{0}\right)}} \frac{\left|(P_{k} p^{1} \mu, P_{k} \overbrace{p^{0} \cdots p^{0}}^{(k-l) \times})_{L^{2}}\right|}{\left\langle r^{1} M_{k} p^{1} \mu, \mu\right\rangle^{\frac{1}{2}}\left\langle M_{l} \nu, \nu\right\rangle^{\frac{1}{2}}} \\
& =\sup _{\substack{0 \neq \tilde{\mu} \in \ell^{2}\left(\Omega_{k-1}^{1}\right) \\
0 \neq \tilde{\nu} \in \ell^{2}\left(\Omega_{l}^{0}\right)}} \frac{\left|\langle\tilde{\mu},\left(r^{1} M_{k} p^{1}\right)^{-\frac{1}{2}} r^{1} M_{k} \overbrace{p^{0} \cdots p^{0}}^{(k-l) \times} M_{l}^{-\frac{1}{2}} \tilde{\nu}\rangle\right|}{\|\tilde{\mu}\|\|\tilde{\nu}\|} .
\end{aligned}
$$

We will now estimate the upper bound for $\theta_{k l}$ from Proposition 3.9 in our onedimensional regular grid case resulting in (9). Define $\bar{p}^{\imath}: \ell^{2}\left(\Omega_{m-1}^{2}\right) \rightarrow \ell^{2}\left(\Omega_{m}^{0}\right)$ by $\left(\bar{p}^{\imath} u\right)(x)=\left\{\begin{array}{cc}u(x), \quad x \in \Omega_{m-1}^{2}, \\ 0, \quad x \in \Omega_{m}^{0} \backslash \Omega_{m-1}^{2}\end{array}\right.$ and $\bar{r}^{\imath}: \ell^{2}\left(\Omega_{m}^{0}\right) \rightarrow \ell^{2}\left(\Omega_{m-1}^{2}\right)$ by $\left(\bar{r}^{\imath} u\right)(x)=u(x)$. Then $\bar{r}^{\imath}=\left(\bar{p}^{\imath}\right)^{*}, p^{\imath}=\frac{1}{2}\left[\begin{array}{lll}(-1)^{\imath} & 2 & (-1)^{\imath}\end{array}\right] \bar{p}^{\imath}$ and $r^{\imath}=\bar{r}^{\imath} \frac{1}{2}\left[\begin{array}{lll}(-1)^{\imath} & 2 & (-1)^{\imath}\end{array}\right]$.

The mass matrix $M_{m}$ is given by the difference stencil $\frac{1}{6} h_{m}\left[\begin{array}{lll}1 & 4 & 1\end{array}\right]$. It satisfies the relation

$$
\left(M_{m}-h_{m} I\right) p^{0}=\frac{1}{4} \bar{p}^{0}\left(M_{m-1}-h_{m-1} I\right) .
$$

Since $r^{1} p^{0}=0$ (use (1)), we obtain

$$
r^{1} M_{k} \underbrace{p^{0} \cdots p^{0}}_{(k-l) \times}=r^{1}\left(M_{k}-h_{k} I\right) \underbrace{p^{0} \cdots p^{0}}_{(k-l) \times}=r^{1}\left(\frac{1}{4}\right)^{k-l} \underbrace{\bar{p}^{0} \cdots \bar{p}^{0}}_{(k-l) \times}\left(M_{l}-h_{l} I\right) .
$$

The set $\left\{\psi_{m}^{(j)}: x \mapsto \sqrt{2 h_{m}} \sin (\pi j x)\right\}_{j \in\left\{1, \ldots, n_{m}\right\}}$, where $n_{m}:=h_{m}^{-1}-1$, forms an orthonormal basis of $\ell^{2}\left(\Omega_{m}^{0}\right)$ that consists of eigenvectors $\psi_{m}^{(j)}$ of $M_{m}$ and $\frac{1}{2}\left[\begin{array}{lll}(-1)^{\imath} & 2 & (-1)^{\imath}\end{array}\right]$ with eigenvalues $\frac{1}{3} h_{m}\left(2+\cos \left(\pi j h_{m}\right)\right)$ and $\left(1+(-1)^{\imath} \cos \left(\pi j h_{m}\right)\right)$, respectively. There holds

$$
\bar{p}^{0} \psi_{m-1}^{(j)}=\frac{1}{2} \sqrt{2}\left(\psi_{m}^{(j)}-\psi_{m}^{\left(n_{m}+1-j\right)}\right) \quad\left(\left\{j \in\left\{1, \ldots, n_{m-1}\right\}\right) .\right.
$$

For $r^{1}, p^{1}$, we have

$$
\bar{r}^{1} \psi_{m}^{(j)}=\bar{r}^{1} \psi_{m}^{\left(n_{m}+1-j\right)}=\left.\psi_{m}^{(j)}\right|_{\Omega_{m-1}^{1}} \text { and } \quad \bar{p}^{1}\left(\left.\psi_{m}^{(j)}\right|_{\Omega_{m-1}^{1}}\right)=\frac{1}{2}\left(\psi_{m}^{(j)}+\psi_{m}^{\left(n_{m}+1-j\right)}\right)
$$




$$
\begin{aligned}
& \left(j \in\left\{1, \ldots, n_{m-1}+1\right\}\right) \text {. From } \\
& \left\langle\left.\psi_{m}^{(j)}\right|_{\Omega_{m-1}^{1}},\left.\psi_{m}^{(i)}\right|_{\Omega_{m-1}^{1}}\right\rangle=\left\langle\bar{r}^{1} \psi_{m}^{(j)}, \bar{r}^{1} \psi_{m}^{(i)}\right\rangle \\
& =\left\langle\frac{1}{2}\left(\psi_{m}^{(j)}+\psi_{m}^{\left(n_{m}+1-j\right)}\right), \psi_{m}^{(i)}\right\rangle= \begin{cases}0, & i \neq j \in\left\{1, \ldots, n_{m-1}+1\right\}, \\
\frac{1}{2}, & i=j \in\left\{1, \ldots, n_{m-1}\right\}, \\
1, & i=j=n_{m-1}+1,\end{cases}
\end{aligned}
$$

we have that $\left\{\left.\sqrt{2} \psi_{m}^{(j)}\right|_{\Omega_{m-1}^{1}}: j \in\left\{1, \ldots, n_{m-1}\right\}\right\} \cup\left\{\left.\psi_{m}^{\left(n_{m-1}+1\right)}\right|_{\Omega_{m-1}^{1}}\right\}$ is an orthonormal basis of $\ell^{2}\left(\Omega_{m-1}^{1}\right)$. Note that $\operatorname{span}\left\{\psi_{m}^{\left(n_{m-1}+1\right)}\right\} \perp \operatorname{range} p^{0}: \ell^{2}\left(\Omega_{m-1}^{0}\right) \rightarrow \ell^{2}\left(\Omega_{m}^{0}\right)$, which means that the different scaling of $\left.\psi_{m}^{\left(n_{m-1}+1\right)}\right|_{\Omega_{m-1}^{1}}$ does not enter our computations.

After a basis transformation to the orthonormal bases of $\ell^{2}\left(\Omega_{l}^{0}\right)$ and $\ell^{2}\left(\Omega_{k}^{1}\right)$, straightforward computations using the relations above now show that for $l=k-1$,

$$
\begin{aligned}
\theta_{k k-1} & \leq\left\|\left(r^{1} M_{k} p^{1}\right)^{-\frac{1}{2}} r^{1} \frac{1}{4} \bar{p}^{0}\left(M_{k-1}-h_{k-1} I\right) M_{k-1}^{-\frac{1}{2}}\right\| \\
& =\max \left\{\frac{x\left(1-x^{2}\right)}{2 \sqrt{1+2 x^{2}}}: x=\cos \left(\pi j h_{k}\right), j \in\left\{1, \ldots, n_{k-1}\right\}\right\} \\
& \leq \eta:=\max \left\{\frac{x\left(1-x^{2}\right)}{2 \sqrt{1+2 x^{2}}}: x \in[0,1]\right\} \approx .153
\end{aligned}
$$

and that for $l<k-1$,

$$
\begin{aligned}
\theta_{k l} \leq & \left\|\left(r^{1} M_{k} p^{1}\right)^{-\frac{1}{2}} r^{1} \frac{1}{4} \bar{p}^{0}\right\|\|\frac{1}{4}^{k-l-1} \underbrace{\bar{p}^{0} \cdots \bar{p}^{0}}_{(k-l-1) \times}\|\left\|\left(M_{l}-h_{l} I\right) M_{l}^{-\frac{1}{2}}\right\| \\
= & \max \left\{\frac{\sqrt{6}|x|}{8 \sqrt{h_{k}}}: x=\cos \left(\pi j h_{k}\right), j \in\left\{1, \ldots, n_{k-1}\right\}\right\} \\
& \cdot_{\frac{1}{4}}^{k-1-l} \cdot \max \left\{\frac{|1-x| \sqrt{h_{l}}}{(2+x) \sqrt{3}}: x=\cos \left(\pi j h_{l}\right), j \in\left\{1, \ldots, n_{l}\right\}\right\} \\
\leq & \frac{\sqrt{6}}{8 \sqrt{h_{k}}} \cdot \frac{1}{4}^{k-1-l} \cdot \frac{2 \sqrt{h_{l}}}{\sqrt{3}}=\sqrt{2}\left(\frac{1}{4} \sqrt{2}\right)^{k-l} .
\end{aligned}
$$

By using Lemma 3.8 and $\rho(\Theta-I) \leq\|\Theta-I\|_{\infty} \leq 2\left(\eta+\sqrt{2} \sum_{n \geq 2}\left(\frac{1}{4} \sqrt{2}\right)^{n}\right) \approx .85<1$, we conclude that (9) is valid.

3.4. An $H^{1}$-equivalent norm on $\mathcal{M}_{J}$. A consequence of (9) is that

$$
\left\|Z_{l}^{(k)}\right\|_{L^{2} \leftarrow L^{2}} \lesssim 1 \quad \text { (uniformly in } k \geq l \text { ). }
$$

Let $Q_{k}: L^{2}(\Omega) \rightarrow \mathcal{M}_{k}$ be the $L^{2}$-orthogonal projection on $\mathcal{M}_{k}$. Then it is well known that

$$
\left\|I-Q_{k}\right\|_{L^{2} \leftarrow H^{1}} \lesssim h_{k}
$$

From the inverse estimate

$$
\|\cdot\|_{H^{1}} \lesssim h_{k}^{-1}\|\cdot\|_{L^{2}} \quad \text { on } \mathcal{M}_{k}
$$

we have

$$
\left\|Q_{k}\right\|_{H^{1} \leftarrow H^{1}} \equiv 1 .
$$


The estimate (14) also implies that for $u \in \mathcal{V}_{k}$,

$$
\begin{aligned}
\|u\|_{L^{2}} & \equiv\|u\|_{\mathcal{M}_{k}}=\left\|\left(I-Y_{k-1}\right) u\right\|_{\mathcal{M}_{k}} \\
& \leq\left\|\left(I-Q_{k-1}\right) u\right\|_{\mathcal{M}_{k}}
\end{aligned}
$$

In $[12$, Appendix], a compact proof is given of

$$
\sum_{k=1}^{J}\left\|\left(Q_{k}-Q_{k-1}\right) u\right\|_{H^{1}}^{2} \bar{\sim}\|u\|_{H^{1}}^{2} \quad\left(u \in \mathcal{M}_{J}\right)
$$

(where $Q_{-1}:=0$ ). As we will see, this proof with $Z_{k}^{(J)}$ playing the role of $Q_{k}-Q_{k-1}$ will also yield (10).

Theorem 3.10. We have $\sum_{k=0}^{J}\left\|Z_{k}^{(J)} u\right\|_{H^{1}}^{2} \bar{\sim}\|u\|_{H^{1}}^{2}\left(u \in \mathcal{M}_{J}\right)$.

Proof (based on [12, Appendix]). Let $u \in \mathcal{M}_{J}$ and $u_{l}=\left(Q_{l}-Q_{l-1}\right) u$. Then $u_{l} \in \mathcal{M}_{l}$, and so for $l<k \leq J$, we have $Z_{k}^{(J)} u_{l}=0$. For $k \leq l$, it follows from (15), (13) and (14) that

$$
\left\|Z_{k}^{(J)} u_{l}\right\|_{H^{1}} \lesssim h_{k}^{-1}\left\|Z_{k}^{(J)} u_{l}\right\|_{L^{2}} \lesssim h_{k}^{-1}\left\|u_{l}\right\|_{L^{2}} \lesssim h_{k}^{-1} h_{l}\left\|u_{l}\right\|_{H^{1}}
$$

Let $l \wedge m=\min \{l, m\}$. Writing $u=\sum_{l=0}^{J} u_{l}$, we get

$$
\begin{aligned}
\sum_{k=0}^{J}\left\|Z_{k}^{(J)} u\right\|_{H^{1}}^{2} & =\sum_{k=0}^{J} \sum_{l, m=k}^{J}\left(Z_{k}^{(J)} u_{l}, Z_{k}^{(J)} u_{m}\right)_{H^{1}}=\sum_{l, m=0}^{J} \sum_{k=0}^{l \wedge m}\left(Z_{k}^{(J)} u_{l}, Z_{k}^{(J)} u_{m}\right)_{H^{1}} \\
& \leq \sum_{l, m=0}^{J} \sum_{k=0}^{l \wedge m} h_{k}^{-2} h_{l} h_{m}\left\|u_{l}\right\|_{H_{1}}\left\|u_{m}\right\|_{H^{1}} \lesssim \sum_{l, m=0}^{J} h_{l \wedge m}^{-2} h_{l} h_{m}\left\|u_{l}\right\|_{H^{1}}\left\|u_{m}\right\|_{H^{1}} \\
& \lesssim \sum_{l=0}^{J}\left\|u_{l}\right\|_{H^{1}}^{2} \equiv\|u\|_{H^{1}}^{2}
\end{aligned}
$$

by (18).

In [12, Lemma 6.1], it was proved that for $k \leq l, u \in \mathcal{M}_{k}$ and $v \in \mathcal{M}_{l}$,

$$
\left|(u, v)_{H^{1}}\right| \lesssim\left(h_{k} h_{l}\right)^{-\frac{1}{2}}\|u\|_{H^{1}}\|v\|_{L^{2}} .
$$

Using this and (17), we obtain for $u \in \mathcal{M}_{J}$

$$
\begin{aligned}
\|u\|_{H^{1}}^{2} & =\sum_{k, l=0}^{J}\left(Z_{k}^{(J)} u, Z_{l}^{(J)} u\right)_{H^{1}} \\
& \lesssim \sum_{k, l=0}^{J}\left(h_{k} h_{l}\right)^{-\frac{1}{2}} \min \left\{h_{k-1}, h_{l-1}\right\}\left\|Z_{k}^{(J)} u\right\|_{H^{1}}\left\|Z_{l}^{(J)} v\right\|_{H^{1}} \lesssim \sum_{k=0}^{J}\left\|Z_{k}^{(J)} u\right\|_{H^{1}}^{2},
\end{aligned}
$$

which completes the proof.

\section{ACKNOWLEDGEMENTS}

The author wishes to acknowledge the hospitality of the Institut für Informatik und Praktische Mathematik, Universität Kiel, where much of this work was performed. Furthermore, he would like to thank Professors W. Hackbusch (Kiel) and M. Dryja (Warsaw) and Dr. A. Reusken (Eindhoven) for fruitful discussions. 


\section{REFERENCES}

1. J.H. Bramble and J.E. Pasciak, New convergence estimates for multigrid algorithms, Math. Comp. 49 (1987), 311-329. MR 89b:65234

2. W. Hackbusch, A new approach to robust multi-grid methods, ICIAM '87: Proceedings of the First International Conference on Industrial and Applied Mathematics (Philadelphia) (J. McKenna and R. Temam, eds.), SIAM, 1988. MR 90f:65193

3. tions, Numer. Math. 56 (1989), 229-245. MR 90i:65212

4. Iterative lösung großer schwachbesetzter gleichungssysteme, B.G. Teubner, Stuttgart, 1991. MR 92i:65002

5. — The frequency decomposition multi-grid method II. Convergence analysis based on the additive Schwarz method, Numer. Math. 63 (1992), 433-453. MR 93j:65203

6. The frequency decomposition multi-grid method, Multigrid Methods IV, Proceedings of the Fourth European Multigrid Conference, Amsterdam, July 6-9, 1993 (Basel) (P.W. Hemker and P. Wesseling, eds.), Birkhäuser, 1994, pp. 43-56. MR 95h:65095

7. J. Junkherr, Multigrid methods for weakly singular integral equations of the first kind, Ph.D. thesis, Christian-Albrechts-Universität Kiel, 1994.

8. W. Mulder, A new multigrid approach to convection problems, J. Comp. Phys. 83 (1989), 303-323. MR 90f: 76013

9. N.H. Naik and J. Van Rosendale, The improved robustness of multigrid elliptic solvers based on multiple semicoarsened grids, SIAM J. Numer. Anal. 30 (1993), 215-229. MR 94b:65159

10. C.W. Oosterlee and P. Wesseling, On the robustness of a multiple semi-coarsened grid method, ZAMM 75 (1995), no. 4, 251-257. CMP 95:12

11. R.P. Stevenson, Robustness of the additive and multiplicative frequency decomposition multilevel method, Computing 54 (1995), 331-346. CMP 95:13

12. J. $\mathrm{Xu}$, Iterative methods by space decomposition and subspace correction, SIAM Rev. 34 (1992), 581-613. MR 93k:65029

13. H. Yserentant, Old and new convergence proofs for multigrid methods, Acta Numerica (1993), 285-326. MR 94i:65128

Department of Mathematics, Nijmegen University, Toernooiveld 1, 6525 ED NiJMEgen, The Netherlands

E-mail address: stevenso@sci.kun.nl 